\title{
Rheology and microrheology of a microstructured fluid: The gellan gum case
}

\author{
M. Caggioni ${ }^{\text {a) }}$ \\ Department of Physics and DEAS, Harvard University, \\ Cambridge, Massachusetts 02138 \\ P. T. Spicer
}

The Procter and Gamble Company, Corporate Engineering, 8256 Union Centre Boulevard, West Chester, Ohio 45069

D. L. Blair

Department of Physics and DEAS, Harvard University, Cambridge, Massachusetts 02138

\section{S. E. Lindberg}

The Procter and Gamble Company, Corporate Engineering, 8256 Union Centre Boulevard, West Chester, Ohio 45069

\author{
D. A. Weitz \\ Department of Physics and DEAS, Harvard University, \\ Cambridge, Massachusetts 02138
}

(Received 27 September 2006; final revision received 1 June 2007)

\begin{abstract}
Synopsis
Particle tracking microrheology is used to study the effect of a constant applied shear during gelation of aqueous gellan gum with a monovalent salt. Shear modifies the gellan gum hydrogel microstructure and the bulk rheological properties of the system, depending on whether shear is applied during gelation or afterwards. The microstructure determines the linear elastic response of the gel, as well as the critical strain and stress above which the response becomes nonlinear. Bulk oscillatory rheology is used to study microstructured gellan gum hydrogels at different polymer and salt concentrations. The similarity between our system and concentrated microgel particle suspensions can be explained by considering the microstructured gellan system to be composed of microgel particles whose size is set by the applied shear stress magnitude during gelation. Polymer concentration and ionic strength control the individual microgel particles' elastic properties. We also find the gellan system exhibits an isoenergetic transition from the jammed to un-jammed state when sheared, similar to jammed colloidal systems [C. G. Robertson and X. R. Wang,
\end{abstract}

\footnotetext{
${ }^{\text {a) }}$ Author to whom correspondence should be addressed; electronic mail: caggioni@deas.harvard.edu
} 
"Isoenergetic jamming transition in particle-filled systems," Phys. Rev. Lett. 95, 075703 (2005)]. (C) 2007 The Society of Rheology. [DOI: 10.1122/1.2751385]

\section{INTRODUCTION}

Gelation under shear is an attractive and technologically useful way to prepare microstructured fluids with tunable rheological properties. Shear during gelation has been shown to modify the elastic properties for various hydrogels [Sworn and Sanderson (1995); Norton et al. (1999); Altmann et al. (2004)] as a result of microstructure modification. However, a clear understanding of the correlation between microstructure and bulk rheological properties is still lacking. Standard techniques used to investigate hydrogel elastic properties, such as oscillatory rheology, are unable to characterize samples at the length scale of the microstructure. Gellan gum hydrogel is a particularly attractive model system as it gels at very low polymer concentration (as low as $\sim 0.01 \% \mathrm{w} / \mathrm{w}$ ) and the gel's transparency allows optical investigation of their properties. Thus, gellan gum appears to be an ideal model system for the study of thermoreversible gelation and the effect of shear on the gelation transition. The amount of shear has been reported to be of critical importance for the determination of the elastic properties of these gels [Sworn $e t$ al. (1995)].

Gellan gum is an extracellular polysaccharide secreted by the microorganism Sphingomonas elodea. Gellan gum forms thermoreversible physical gels at low concentrations when hot solutions are cooled in the presence of gel-promoting cations. As the hot solution cools, gellan gum undergoes a disorder-order coil-helix transition [Ogawa et al. (2005)]. Gel-promoting cations such as sodium, potassium, calcium, and magnesium promote aggregation of the gellan double helices to form a three-dimensional network [Ohtsuka and Watanabe (1996)].

Our goal is to clarify the role of shear, applied during gelation, on the final rheological properties of the gel. To this aim, we vary preparation procedures to produce two different gel types: quiescently cooled gels (QC), in which no shear is applied during gelation; and shear cooled gels (SC), in which a constant shear rate is applied during gelation. Linear oscillatory rheology is used to compare the elastic properties of these two systems. We find a stable and reproducible difference in their linear elastic response. We also find a clear difference between their yielding behavior: while the quiescently cooled gel yields sharply and then recovers slowly, the shear cooled gel demonstrates a smoother transition from elastic-like to fluid-like behavior and a faster recovery of its original elastic properties after yielding. The structural differences between the two systems are studied by optical microscopy observations using a rotating shear stage to characterize the native gel structure and its yielding behavior. Multi particle tracking microrheology is used to measure the local elastic modulus and probe its heterogeneities.

\section{MATERIALS AND METHODS}

\section{A. Sample preparation}

Gellan gum, Kelcogel (CP Kelko, San Diego, CA) powder is added to de-ionized water at room temperature and the mixture is heated to $80{ }^{\circ} \mathrm{C}$ under constant agitation to dissolve the powder. After $10 \mathrm{~min}$ at $80{ }^{\circ} \mathrm{C}$, a $1 \mathrm{M} \mathrm{NaCl}$ solution is added to the polymer dispersion. No polymer aggregation is observed upon addition of the salt solution at high temperature. Gelation is induced by lowering the temperature of the system. A fixed cooling rate of $0.5 \mathrm{~K} / \mathrm{min}$ is used to cool the sample from 80 to $25^{\circ} \mathrm{C}$. The cooling rate is chosen in order to maximize the reproducibility of the sample preparation; this parameter is reported not to be critical in similar studies of the gelation properties of different 
biopolymers [Norton et al. (1999)]. The samples are prepared in two ways: quiescently cooled samples (QC) are obtained by cooling the system with no applied shear while sheared cooled samples (SC) are cooled while applying a constant applied shear rate of $100 \mathrm{~s}^{-1}$. The sol-gel transition for all the samples studied is above $32{ }^{\circ} \mathrm{C}$ and depends slightly on the concentration of salt.

\section{B. Oscillatory Rheology measurements}

Bulk oscillatory rheological measurements are performed with a Bohlin C-VOR rheometer (Malvern instrument UK) using a cone and plate geometry with 40-mm-diam, $4^{\circ}$ cone slope, and a minimum gap of $0.15 \mathrm{~mm}$. A thin layer of silicon oil (viscosity $20 \mathrm{cP}$ ) is placed around the sample to avoid water evaporation during the tests. Data are collected every four oscillation cycles. Most of the data presented in this study are from dynamic strain sweep tests at a fixed oscillation frequency of $1 \mathrm{~Hz}$. The cone and plate measurements matched results obtained using parallel plate and concentric cylinder geometries, indicating wall slip does not bias these measurements.

\section{Multi particle tracking microrheology}

Surfactant-free polystyrene particles of $1 \mu \mathrm{m}$ diameter (Molecular Probes, U.S.) are introduced as tracers during the preparation of the gel. The tracer particles are negatively charged at neutral $p \mathrm{H}$ and their interaction with the gellan polymer is repulsive. At the tracer concentrations used we see no difference in the gel bulk rheology with or without tracers, indicating tracer interaction with the gellan does not affect the microstructure. This interaction has to be considered when we interpret the Brownian motion of the tracers since polymer depletion can increase tracer mobility. Tracers are imaged with a $63 \times$ objective on a Leica DM IRB inverted microscope on a Newport Vibration Isolation Workstation. The sample is placed in a $200-\mu$ m-thick optical cell [Valentine et al. (2001)], allowing imaging of about 100 probe particles in a focal plane in the middle of the cell $100 \mu \mathrm{m}$ from each wall. Video images of the particles undergoing Brownian motion are collected with a Phantom V5 camera (Vision Research, U.S.) using a temporal resolution up to $3000 \mathrm{frames} / \mathrm{s}$ and an exposure time of $50 \mu \mathrm{s}$. In each frame the tracer position is identified by finding the brightness-averaged centroid position which allows us to improve our pixel resolution $(0.25 \mu \mathrm{m} / \mathrm{px})$ to a subpixel resolution of approximately $10 \mathrm{~nm}$ [Crocker and Grier (1996)]. In order to obtain data over a wide rage of lag times and thus frequencies, for each microrheology experiment we acquire two different image sequences: 8000 frames at a frame rate of 3000 frames/s and 8000 frames at $30 \mathrm{frames} / \mathrm{s}$. We independently analyze the two image sequences for each experiment. Comparison of the results in the overlapping range of lag times also provides a way to check for error in the tracking procedure or artifacts due to convection.

\section{RESULTS}

\section{A. Effect of shear during gelation on gel microstructure}

The structural modifications of the hydrogels, their stability, and the relationship to the applied shear during the sol-gel transition are studied using two gelation procedures. In the first experiment quiescently cooled (QC) samples are prepared at $0.75 \% \mathrm{w} / \mathrm{w}$ polymer in $10 \mathrm{mM} \mathrm{NaCl}$ and $0.1 \% \mathrm{w} / \mathrm{w}$ polymer in $100 \mathrm{mM} \mathrm{NaCl}$. At low strain, the linear viscoelastic response of QC samples is dominated by the elastic component up to a strain of $\sim 1$, whereupon the system begins to yield and respond nonlinearly with the viscous component dominating $\left(G^{\prime \prime}>G^{\prime}\right)$ as shown in Figs. 1(a) and 1(c). The recovery of the 

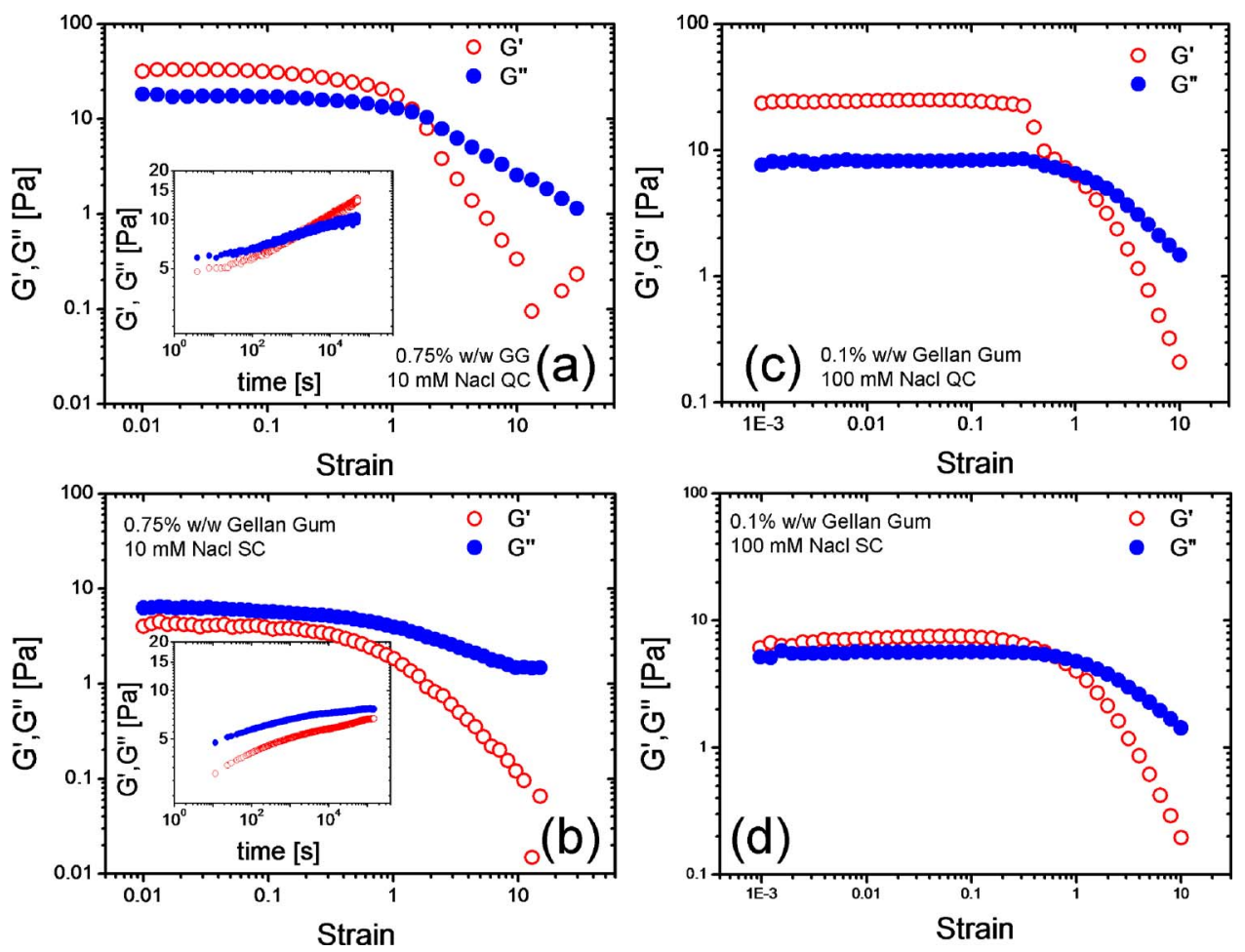

FIG. 1. Comparison between quiescently cooled QC (a-c) and sheared cooled SC (b-d) gellan gum gel with $0.75 \% \mathrm{w} / \mathrm{w}$ polymer fraction in $10 \mathrm{mM} \mathrm{NaCl}$ water solution $(\mathrm{a}-\mathrm{b})$ and gellan gum gel with $0.1 \% \mathrm{w} / \mathrm{w}$ polymer fraction in $100 \mathrm{mM} \mathrm{NaCl}$ water solution $(\mathrm{c}, \mathrm{d})$. We report the results of a strain sweep in the oscillatory rheology experiment with cone and plate geometry at a fixed frequency of $1 \mathrm{~Hz}$ for the different samples. In the plots the elastic modulus is indicated by the open circles and the viscous modulus by the solid circles. (a)-(c) At low strain $G^{\prime}>G^{\prime \prime}$ for the QC sample but the moduli values are higher than for the SC sample. (a) The inset shows the evolution of the sample elastic properties after yielding. The moduli are measured by applying a small oscillatory strain of $1 \%$ at a frequency of $1 \mathrm{~Hz}$. After $12 \mathrm{~h}$ the QC sample has not yet recovered its original values but $G^{\prime}$ is higher than $G^{\prime \prime}$. (b) At low strain $G^{\prime \prime}>G^{\prime}$ for the SC sample. In the inset we show the evolution of the elastic properties after the strain sweep. The sample slowly ages but the elastic modulus never grows higher than the viscous modulus as in the case of the QC sample (a). (d) The SC sample has lower moduli than the QC sample.

elastic properties after yielding is monitored by applying a small oscillatory deformation (strain 1\%) well below the yield strain at a fixed frequency of $1 \mathrm{~Hz}$. The recovery of the initial properties is very slow for the QC sample and the elastic behavior $\left(G^{\prime}>G^{\prime \prime}\right)$ returns only after several hours, as shown in the inset of Fig. 1(a).

The same experiment performed on the SC samples with the same compositions $(0.75 \% \mathrm{w} / \mathrm{w}$ polymer in $10 \mathrm{mM}$ aqueous $\mathrm{NaCl}$ solution and $0.1 \% \mathrm{w} / \mathrm{w}$ polymer in $100 \mathrm{mM}$ aqueous $\mathrm{NaCl}$ solution) shows different moduli in the linear regime. The $0.75 \%$ SC sample shows viscous-dominated behavior in the linear-response region but starts to respond nonlinearly at a strain of $\sim 0.5$ as shown in Fig. 1(b). The $0.1 \%$ SC sample exhibits a lower elastic modulus [Fig. 1(d)] than the QC preparation in Fig. 1(c) and its yielding behavior is not nearly as sharp as the QC sample in Fig. 1(c). The long-time evolution of the postyield elastic properties in the $0.75 \% \mathrm{SC}$ sample exhibits a slow increase of both moduli, but never exhibits elastic behavior [Fig. 1(b) inset]. These experiments demonstrate the intrinsic difference between the QC and SC samples both for the linear elastic properties and the yielding behavior. It is not possible to obtain a SC 
system by shearing a QC one. Even shearing a QC sample for several minutes at a strain rate of $100 \mathrm{~s}^{-1}$ never produces a sample with the same rheological properties as the SC. The sample compositions used in the preceding experiments demonstrate the distinct differences brought about by shear in the strain sweep and time evolution of the moduli, as well as the importance of ionic strength to the gel properties. At high polymer concentration $(0.75 \% \mathrm{w} / \mathrm{w})$ the shear applied during preparation produces a switch between elastic and viscous dominance at the probing frequency. Comparison between the two sample compositions also indicates that similar elastic properties can be obtained for very different polymer concentration by tuning the ionic strength since cations act as "crosslinkers" in the gelation process [Ohtsuka and Watanabe (1996)].

The preparation method strongly affects the final properties of the gel and the recovery after large strain, meaning the process used to prepare gellan-based products is of critical importance and will affect stability, aesthetics, and variability in any product containing gellan gum. In order to relate these observations to differences in gel microstructure [Altmann et al. (2004)], QC and SC samples are prepared in a temperature-controlled microscope shear stage (Linkam CSS450), allowing direct study of the sample microstructure before and after yielding.

The samples studied in these experiments are prepared with a polymer concentration of $0.05 \% \mathrm{w} / \mathrm{w}$ and a salt concentration of $100 \mathrm{mM} \mathrm{NaCl}$. Samples prepared following both QC and SC procedures show dominantly elastic behavior. Fluorescent colloidal particles with diameter of $0.5 \mu \mathrm{m}$ are dispersed in the system at high temperature before gelation to allow imaging of the heterogeneous gel microstructure. Observation of a QC sample after preparation in the microscope shear stage with a gap of $200 \mu \mathrm{m}$ reveals fluorescent colloidal particles homogeneously dispersed and trapped within the polymeric network [Fig. 2(a)]. After application of a constant shear of $100 \mathrm{~s}^{-1}$ for $1 \mathrm{~min}$, the sample exhibits heterogeneous fracture planes at very large length scales $\sim 1 \mathrm{~mm}$. The colloidal particles in the brighter regions of more concentrated polymer are trapped and highly constrained, whereas in the darker regions they diffuse freely [Fig. 2(b)]. Apparently, shearing a QC sample fractures the gel structure and produces large heterogeneities, thus explaining the very slow recovery found after the strain sweep experiment of a QC sample [Fig. 1(a) inset]. A SC sample prepared in the microscope shear stage [Fig. 2(c)] exhibits no evident differences before shear when compared to the QC one [Fig. 2(a)], with the tracers trapped and homogeneously dispersed in the gel. The main difference appears after shearing the samples for $1 \mathrm{~min}$ at $100 \mathrm{~s}^{-1}$ : unlike the QC sample, the SC sample does not show any microstructural changes when subjected to shear [Fig. 2(d)]. This observation supports the idea that the SC system possesses stable structural heterogeneities formed during the gelation under shear, allowing smooth yielding and preventing breakdown of the microstructure.

\section{B. Multi-particle tracking microrheology}

To gain a more quantitative understanding of the local elasticity and heterogeneities of these systems, multi-particle tracking microrheology (MPTM) is used. This technique entails the simultaneous imaging of the Brownian motion of colloidal tracer particles embedded in the system, providing a measurement of the local viscoelastic response. It provides unique insight into microstructural heterogeneities [Valentine et al. (2001); Oppong et al. (2006)] as it provides a local measure of rheological properties on micron length scales. Three different methods are used to incorporate the tracers into the gel microstructure. For the QC sample, the particles are added above $80{ }^{\circ} \mathrm{C}$, before gelation has occurred, and the sample cooled inside the optical cell. A sample prepared by this 


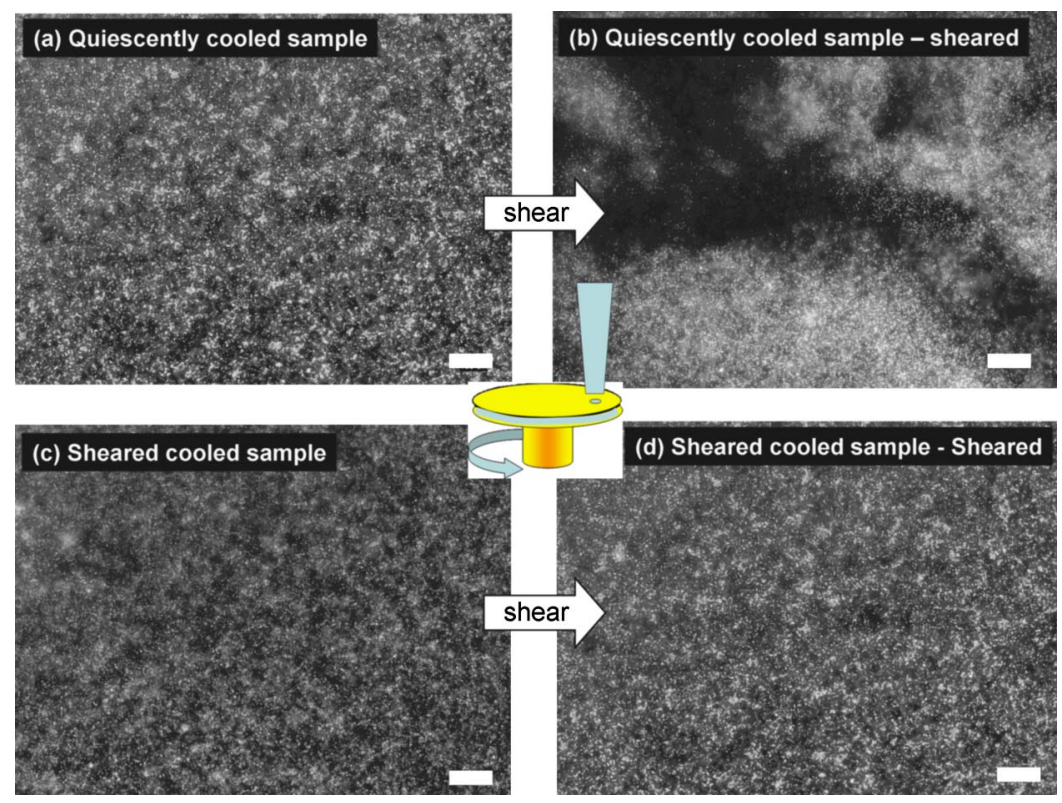

FIG. 2. We can visualize the microstructure present in the system using fluorescent imaging of 0.5 - $\mu \mathrm{m}$-diam fluorescent colloidal tracers. The composition of the samples shown is $0.05 \% \mathrm{w} / \mathrm{w}$ gellan gum in $100 \mathrm{mM} \mathrm{NaCl}$ water solution. The sample is placed in a Linkam shear stage which allows us to control its temperature and to apply shear while observing it through an eccentric window. (a) Image of the sample after quiescently cooling (QC) in the shear stage from 80 to $25^{\circ} \mathrm{C}$. The fluorescent tracer particles appear homogeneously trapped in the gel network. (b) After application of constant shear of $100 \mathrm{~s}^{-1}$ for $1 \mathrm{~min}$ the QC sample shows heterogeneities on large length scales $\sim 1 \mathrm{~mm}$. The tracer particles are trapped within the regions of higher polymer concentration and appear brighter. In the regions of low polymer concentration the particles are free to diffuse. (c) Same sample composition as in (a) but cooled from 80 to $25^{\circ} \mathrm{C}$ in the shear stage under constant applied shear of $100 \mathrm{~s}^{-1}$ (SC procedure). The tracer particles are trapped in the gel network. (d) When the sheared cooled sample (c) is sheared $\left(100 \mathrm{~s}^{-1}\right.$ for $\left.1 \mathrm{~min}\right)$ we notice no changes in the structure.

procedure is denoted hot mixed and quiescently cooled (HM-QC). Colloidal particles cannot be added to a QC sample after the preparation because the mixing would modify the gel structure, compromising the measurement. For the SC sample the colloidal tracers can be added at high $\left(\sim 80^{\circ} \mathrm{C}\right)$ or low $\left(\sim 25^{\circ} \mathrm{C}\right)$ temperature, before or after gelation has occurred, where the first case is referred to as hot mixed and shear cooled (HM-SC) and the second cold mixed and shear cooled (CM-SC). The importance of the mixing procedures is highlighted by a macroscopic observation of the behavior of particles suspended in these systems. Results of a sedimentation study of samples at $0.05 \%$ polymer concentration and $100 \mathrm{mM} \mathrm{NaCl}$ concentration after 2 months reveal that the HM-QC sample [Fig. 3(a)] keeps particles in suspension better than the HM-SC sample, where after two months, there is a higher particle density at the bottom of the vial although particles remain suspended [Fig. 3(b)]. The CM-SC sample shows the highest degree of sedimentation; almost no particles can be observed at the top of the sample after two months [Fig. 3(c)]. The MPTM tracer mean square displacement results for the three systems explain the differences found in their suspension properties (Fig. 3, right). The HM-QC [Fig. 3(a)] and the HM-SC [Fig. 3(b)] samples show a similar diffusive behavior for short lag time $\left(<10^{-2} \mathrm{~s}\right)$ and a plateau at large lag time, indicating that all the particles are trapped within the gel network. This explains the high stability of the particles in the sedimentation study. The mean square displacement of the colloidal tracers in the CM-SC sample 
(a) Hot mixed
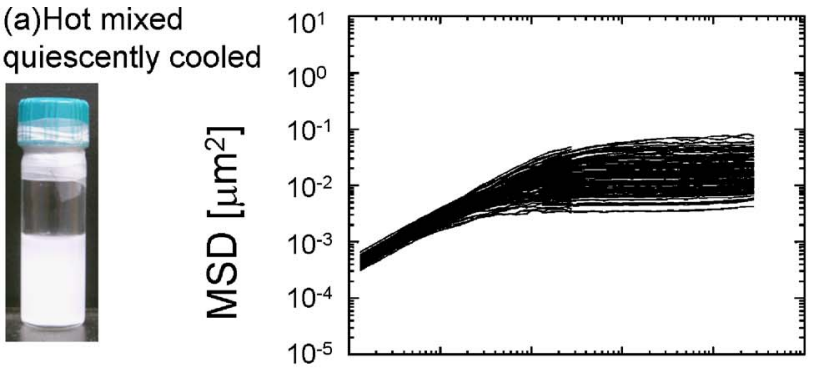

(b) Hot mixed Sheared cooled
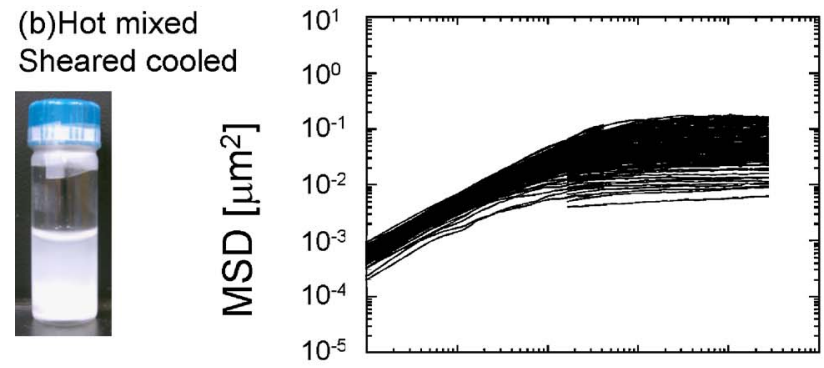

(c)Cold mixed Sheared cooled
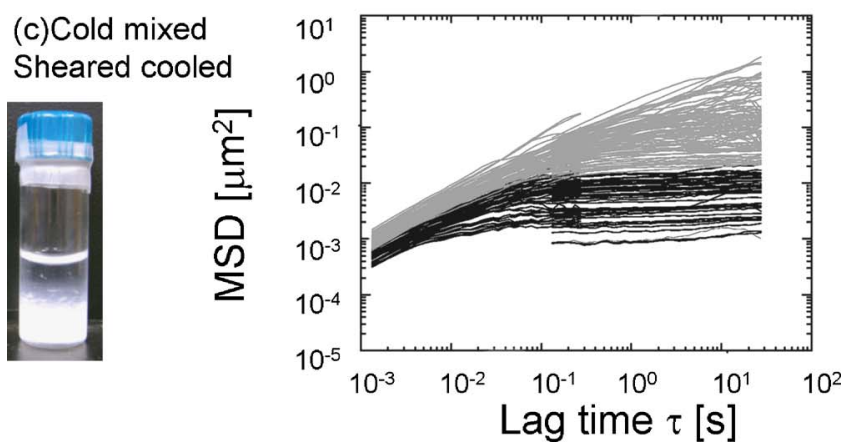

FIG. 3. Left pictures: Two month sedimentation study in samples at $0.05 \%$ weight fraction gellan gum in $100 \mathrm{mM} \mathrm{NaCl}$ water solution. Right plots: multi-particle tracking results showing the mean square displacement of $1 \mu \mathrm{m}$ tracer particles as a function of the lag time $\tau$. (a) Sample prepared incorporating the colloidal tracer at high temperature $\left(80^{\circ} \mathrm{C}\right)$ when the sample is in the sol state and cooling without applied shear: HM-QC. The picture on the left shows no sedimentation. The mean square displacement measured in the microrheology experiment reveals a plateau at long lag time for every particle tracked, indicating all particles are trapped in the gel network. (b) Sample prepared by mixing the colloidal tracer at high temperature and then cooling the sample with constant shear rate applied: HM-SC. The picture shows good long-term sedimentation stability. Microrheology data cannot be distinguished from case a. (c) Sample prepared by cooling with constant applied shear and adding the colloidal tracers at low temperature $\left(25^{\circ} \mathrm{C}\right)$ after gelation: $\mathrm{CM}-\mathrm{SC}$. This sample's bulk properties cannot be distinguished by bulk oscillatory rheology from case (b) and shows colloidal particle sedimentation (picture). Microrheology results reveal the difference between case b and c: two distinct colloidal microenvironments of the tracked particles. About $50 \%$ of the particles show diffusive behavior (no plateau) even at long lag times (cluster 2) while the remaining particles are trapped in the gel network and cannot diffuse at long lag times (cluster 1).

[Fig. 3(c)] shows evident differences when compared to the previous ones. By contrast to the other samples, the mean square displacement of many tracers in the CM-SC sample does not show a plateau at high values of $\tau$. We can also distinguish two populations of tracer particles within the same sample: the ones whose mean square displacement is plotted in black are trapped within the gel network while in gray are the particles that show diffusive behavior even for long lag times. To partition the particle trajectories into clusters we utilize the procedure described in Valentine et al. (2001) for the case of 
agarose gel. We use $F$ statistics on the displacement distribution for lag times of 0.1 and $1 \mathrm{~s}$ to probe the statistical independence of particle trajectories particles in different clusters. We furthermore require the trajectories to be statistically indistinguishable (with a confidence limit of $95 \%$ ) in order to consider them in the same cluster. We find a natural partitioning of this system into two clusters, since it is the maximum number that meets the two conditions just described. For samples HM-QC and HM-SC all the trajectories fit in one single cluster, meaning all tracers experience the same viscoelastic environment.

Using the generalized Stokes-Einstein relation, we interpret the cluster averaged mean square displacement to obtain a good approximation of the local viscoelasticity [Mason (2000)]. For samples HM-QC and HM-SC the viscoelastic moduli results are very similar: at $0.2 \mathrm{~Hz} G^{\prime} \sim 0.1 \mathrm{~Pa}$ for sample HM-QC [Fig. 4(a)] and $G^{\prime} \sim 0.09$ for sample HM-SC [Fig. 4(b)]. For both samples we extract the microrheological properties by averaging the mean square displacement over the entire set of particle trajectories since they can be grouped into a single cluster. The CM-SC case is better described by assuming two independent populations. We show the viscoelastic moduli for the two different cluster groups and we find that cluster 1, composed of the more confined particles, has viscoelastic properties similar to the HM-QC and HM-SC samples with a $G^{\prime} \sim 0.2 \mathrm{~Pa}$ at $0.2 \mathrm{~Hz}$ [Fig. 4(c)]. Cluster 2, containing the less-confined tracers, has an elastic modulus of $G^{\prime} \sim 0.01 \mathrm{~Pa}$ at $0.2 \mathrm{~Hz}$ [Fig. 4(c)]. In order to compare the microrheological results with the ones obtained by means of standard oscillatory rheology, we performed a frequency sweep on the samples used for the microrheological study. Bulk rheology detects significant differences between samples HM-QC and HM-SC [Figs. 4(a) and 4(b)] in contrast with microrheology which did not detect appreciable differences between these two samples. If we compare results for sample HM-SC and CM-SC we observe the opposite situation, microrheology detects rheological differences that are not identified by bulk rheology. For all samples microrheology underestimates the moduli and we believe this difference results from electrostatic interactions between the tracers and the gellan. As both tracers and gel are negatively charged, it is plausible that a depletion layer forms around the tracers, increasing their mobility. This behavior has been observed for other biopolymers [Liu et al. (2006)] using the same tracer particles.

A simple schematic representation of the presumed gel structure with colloidal tracers represented in black and the gel network drawn as a grid is shown on the right side of Fig. 4. The sedimentation study and the microrheology experiment suggest an interpretation of the HM-QC sample as a very large gel structure that traps the tracer particles [Fig. 4(a)]. In the case of HM-SC and CM-SC gelation under shear favors the formation of a heterogeneous gel network on a length scale of $\sim 10-100 \mu \mathrm{m}$. The different results of the sedimentation study and the MPTM experiment for these two cases are interpreted not as the result of a different microstructure but as the effect of the mixing procedure. When tracer particles are added before gelation has occurred (HM-SC), the colloidal tracers are uniformly embedded into high density polymer regions [Fig. 4(b)] while addition of tracer after gelation (CM-SC) prevents such uniform inclusion [Fig. 4(c)]. It is also important to note that while oscillatory rheology can distinguish between samples cooled with and without shear, microrheology cannot. By contrast, the tracer particle order of addition cannot be distinguished by bulk rheology but is evident from the sedimentation study and the MPTM experiments.

Though still not unequivocal, the information provided by MPTM supports the hypothesis of a stable microstructure in the SC gels and leads us to interpret these systems as a concentrated dispersion of microgel particles instead of a single percolating network like the QC case. 


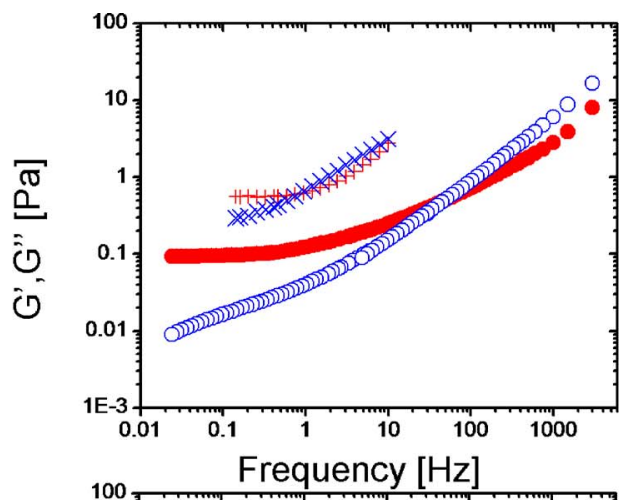

\section{(a)Hot mixed quiescently cooled}
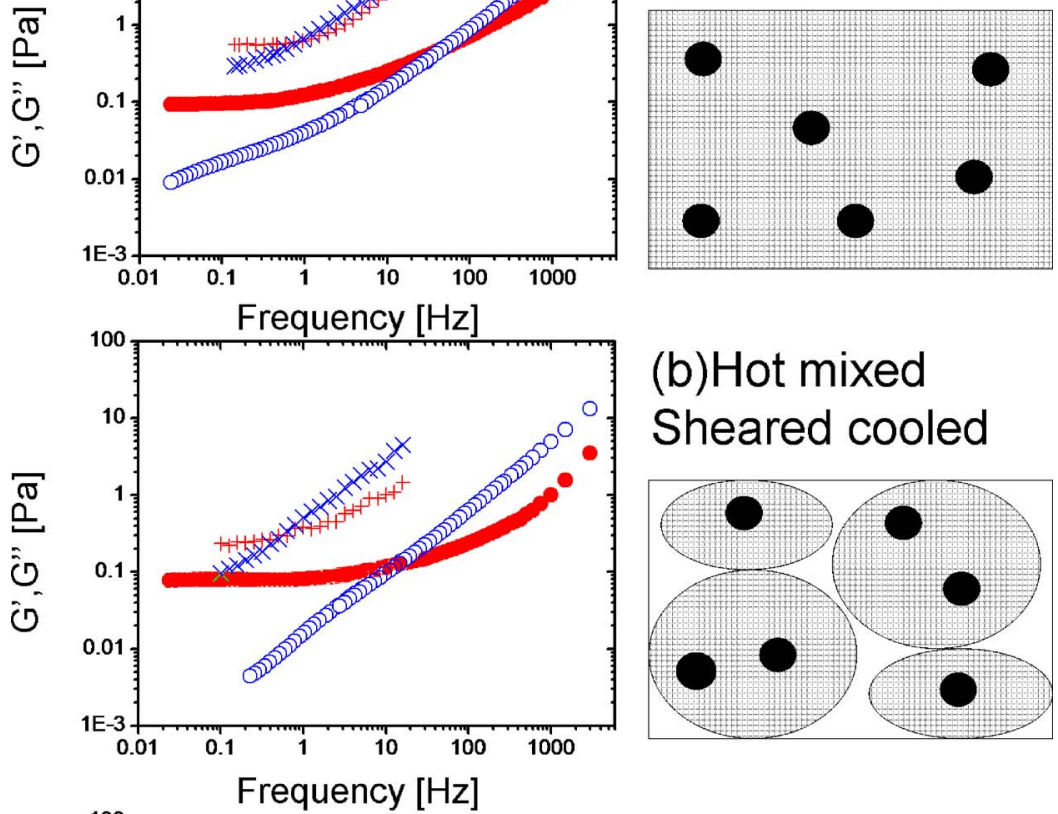

\section{(b)Hot mixed Sheared cooled}
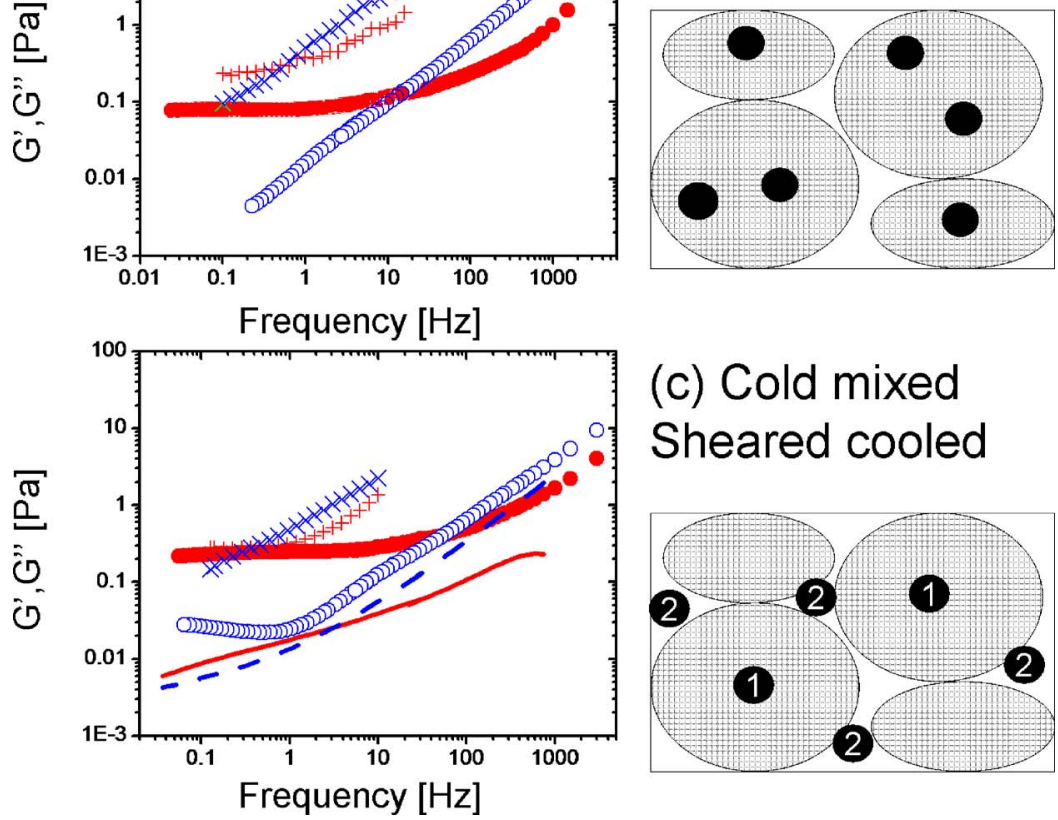

\section{(c) Cold mixed Sheared cooled}

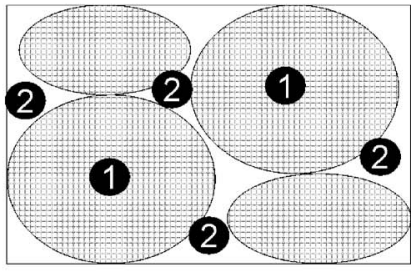

FIG. 4. Right: Schematic description of the microstructure present in the system in the different cases in Fig. 3. We indicate in black the colloidal tracer. Grid areas represent the gel. (a) In the hot mixed and quiescently cooled case (HM-QC) the particles are strictly confined, the trajectories are localized, and the gel structure is homogeneous. (b) Shear during gelation induces heterogeneities in the system; the gel exists as microgel particles at high volume fraction. In the hot mixing procedure the colloidal particles have high probability of being trapped inside the microgel. (c) The microstructure of the gel is similar to the sample (b) since the same shear is applied during gelation. The introduction of colloidal particles in the already structured liquid reduces the likelihood of incorporation inside the microgel structures. Many colloidal tracers show diffusive behavior. Left: comparison between viscoelastic moduli as a function of frequency obtained with microrheology and bulk rheology. In the plot symbols "+" refers to $G^{\prime}$ and " $\times$ " to $G^{\prime \prime}$ obtained with bulk oscillatory rheology. Microrheology results are indicated with solid circle $\left(G^{\prime}\right)$ and open circle $\left(G^{\prime \prime}\right)$. In case (c) microrheological results for the high mobility cluster 2 are indicated by a solid line $\left(G^{\prime}\right)$ and dashed line $\left(G^{\prime \prime}\right)$. Solid circles $\left(G^{\prime}\right)$ and open circles $\left(G^{\prime \prime}\right)$ refer to cluster 1 results. 


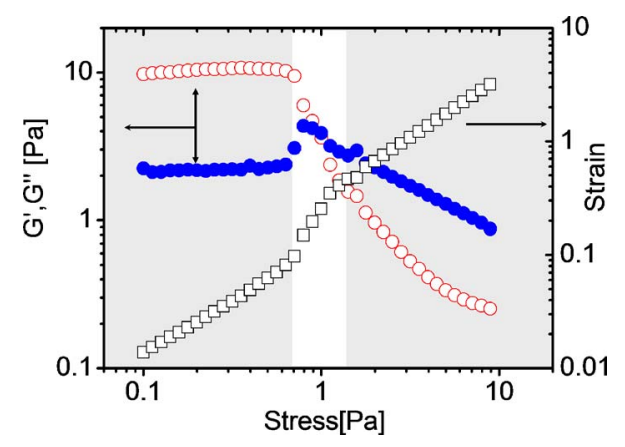

FIG. 5. Left axis: Elastic modulus $G^{\prime}$ (open circles) and viscous modulus $G^{\prime \prime}$ (solid circles) as a function of the maximum amplitude of oscillatory stress applied at fixed frequency of $1 \mathrm{~Hz}$ for a SC sample at $0.2 \%$ weight fraction of gellan gum in $200 \mathrm{Mm} \mathrm{NaCl}$-water solution. Right axis: amplitude of the resulting strain (open squares) as a function of the amplitude of applied stress. The shaded region on the left indicates the range of stresses for which the system responds linearly. At a critical stress of $\sim 0.7 \mathrm{~Pa}$ the system yields and the strain response start to be nonlinear. For stresses higher than $\sim 1 \mathrm{~Pa}$ the strain follows power law scaling with the applied stress (right shaded region).

\section{Oscillatory rheology: Investigating the properties of sheared cooled samples}

Given the MPTM results and the structural differences between QC and SC samples, it is interesting to address the question of how the microstructure affects the macroscopic rheological properties at different polymer and salt concentrations. In order to obtain information on the linear response $\left(G^{\prime}\right.$ and $\left.G^{\prime \prime}\right)$ and on the critical yield stress $\sigma_{y}$ and yield strain $\gamma_{y}$ we perform a strain sweep immediately after preparation of the sample in the rheometer. Three different regions are evident: for low deformation we find a linear response region where the amplitude of the applied stress is directly proportional to the amplitude of the resulting strain and the sample shows dominance of the elastic modulus $\left(G^{\prime}>G^{\prime \prime}\right)$. For higher stresses the elastic modulus decreases and the viscous modulus increases [Miyazaki et al. (2006)] as the system starts to show a nonlinear elastic response. For even higher stresses the strain scales as a power law with the applied stress and the sample displays a predominantly viscous behavior $\left(G^{\prime \prime}>G^{\prime}\right)$. We report in Fig. 5 results for measurement of a SC sample with $0.2 \% \mathrm{w} / \mathrm{w}$ polymer and $200 \mathrm{mM} \mathrm{NaCl}$ salt concentration. The left axis refers to the open circles (elastic modulus $G^{\prime}$ ) and to the solid circles (viscous modulus $G^{\prime \prime}$ ) as a function of the applied stress, while the right axis refers to the value of the strain, indicated by the open squares. At low deformation the moduli demonstrate the linear elastic response of the system. Defining the critical stress $\sigma_{y}$ as the stress at which the strain deviates more than $10 \%$, we characterize the sample yield properties using $\sigma_{y}$ and the critical strain $\gamma_{y}$.

For samples at fixed salt concentration, the linear elastic properties $\left(G^{\prime}, G^{\prime \prime}\right)$ scale with polymer concentration following a power law, $G^{\prime} \propto \phi^{a}$ with $a=2.04 \pm 0.07$ and $G^{\prime \prime} \propto \phi^{b}$ with $b=1.98 \pm 0.08$ (Fig. 6). At fixed salt concentration the critical yield stress $\sigma_{y}$ and strain $\gamma_{y}$ show power law scaling as a function of polymer concentration. As the polymer concentration is increased, $\sigma_{y}$ scales directly $\left(\sigma_{y} \propto \phi^{c}\right.$ with $\left.c=1.04 \pm 0.11\right)$ while $\gamma_{y}$ scales inversely with the polymer concentration $\left(\sigma_{y} \propto \phi^{d}\right.$ with $\left.d=-1.04 \pm 0.07\right)$. Such scaling of $\sigma_{y}$ and $\gamma_{y}$ makes their product invariant with polymer concentration. We will discuss the implication of this finding in the next section. The critical parameters $\sigma_{y}$ and $\gamma_{y}$ can also be measured by monitoring the amplitude of the strain as a function of an increasing steady stress applied to the sample. At low stress a linear increase of the strain with stress 


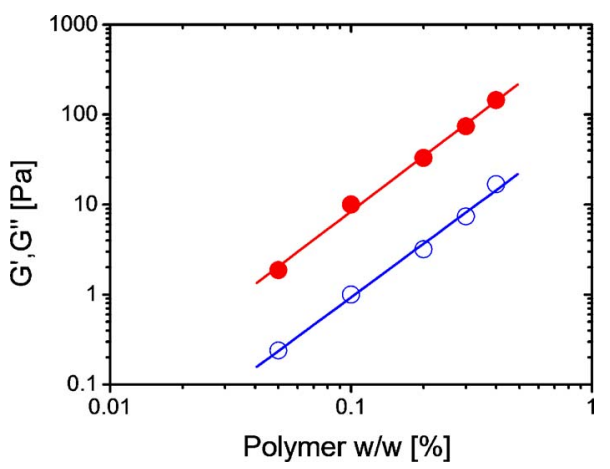

FIG. 6. Scaling of the elastic modulus $G^{\prime}$ (solid circles) and viscous modulus $G^{\prime \prime}$ (open circles) as a function of the polymer weight fraction for a set of SC samples at fixed salt concentration of $200 \mathrm{mM} \mathrm{NaCl}$. Lines represent the best fit of $G^{\prime}$ and $G^{\prime \prime}$ vs polymer weight fraction with a power law fit to $y=a x^{\mathrm{b}}$. For $G^{\prime} a$ $=2.96 \pm 0.06, b=2.04 \pm 0.07$ and for $G^{\prime \prime}$ fit $a=1.95 \pm 0.07, b=1.98 \pm 0.08$.

is observed up to the point where the sample yields and the strain increases rapidly (lower plot of Fig. 7). Good agreement is found between the values of $\sigma_{y}$ and $\gamma_{y}$ in this experiment and the values found with oscillatory-stress measurements. When the experiments are repeated using different salt concentrations, 100 and $300 \mathrm{mM} \mathrm{NaCl}$, the same scaling of $\sigma_{y}$ and $\gamma_{y}$ is found (Fig. 8).

\section{DISCUSSION}

The experiments demonstrate that constant shear applied during gellan gum gelation produces a stable modification of the rheological properties of the final system. Such modification can be described stressing three important differences between the SC and the QC systems:

1. Samples gelled under shear exhibit smaller viscoelastic moduli than samples prepared by quiescent gelation.

2. Systems gelled under shear yield more gradually and in a more reproducible way than those gelled quiescently. The yielding of quiescently gelled samples is very sharp and less reproducible.

3. The effect of shear during the gelation process cannot be reproduced by shear applied after gelation has occurred. It is not possible to obtain a SC sample by just shearing a QC one.

The results agree with a model in which applied shear forms microgel particles whose stiffness depends on salt and polymer concentration. The microstructure is stable if samples are kept below the gel melting temperature to avoid particle coalescence. Microgel particle size can be of order 1-100 $\mu \mathrm{m}$ depending on the magnitude of the shear applied during gelation [Norton et al. (1999)]. Microrheology results are compatible with the existence of the microgel domains. Furthermore, the microrheology of a single microgel particle does not differ from that of the bulk gel prepared by quiescent cooling, suggesting that shear during gelation mainly affects the colloidal-scale structure of the system and not the degree of molecular organization. Comparison of the tracer mean square displacements for the QC case [Fig. 3(a)] and the SC cases [Fig. 3(b)] shows no significant differences, indicating that the local elastic environment of the trapped probe particles is the same in the QC and SC systems. 


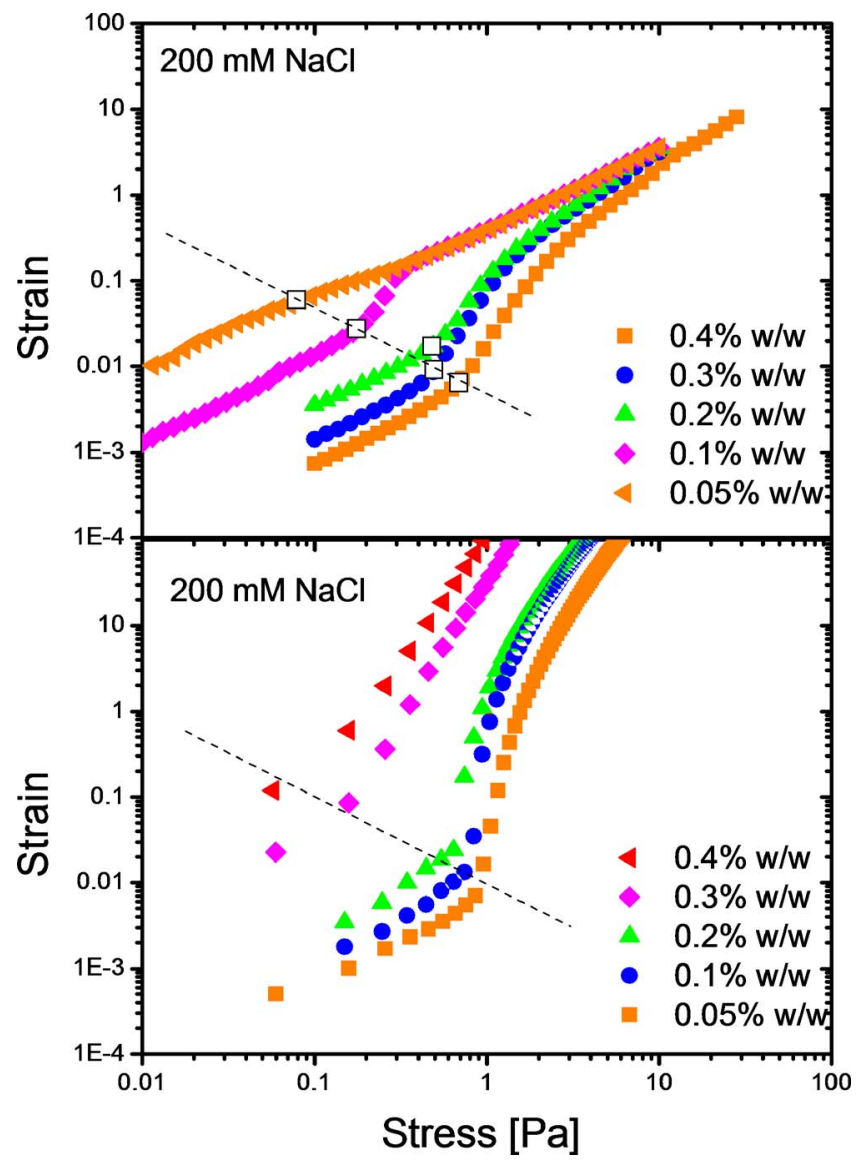

FIG. 7. Upper plot: amplitude of strain response as a function of the oscillatory stress amplitude applied at a fixed frequency of $1 \mathrm{~Hz}$. All curves refer to gellan gum SC samples prepared in $200 \mathrm{mM} \mathrm{NaCl}$-water solution. Different curves refer to different polymer concentration: left triangles $0.4 \%$ polymer weight fraction, diamonds $0.3 \%$, triangles $0.2 \%$, circles $0.1 \%$ and squares $0.05 \%$. Open squares represent the determined critical parameters $\sigma_{y}$ and $\gamma_{y}$. The dashed line is the best hyperbolic fit to the points defined by the yield stress and the yield strain. Lower plot: strain as a function of increasing applied steady stress. We interpret the yield stress as the maximum stress before the strain response becomes nonlinear. The different curves indicate different polymer concentrations in $200 \mathrm{mM} \mathrm{NaCl}$-water solution. Symbols code as in upper plot. The two experiments show good agreement for the determination of critical strain and stress.

Interpretation of the rheological characterization performed on SC samples with different polymer concentration and ionic strength poses a larger challenge. Power law scaling is found for both linear and nonlinear rheological properties as a function of polymer concentration.

We can summarize these scaling relationships for fixed ionic strength as follows:

1. The elastic and viscous modulus increase with the polymer concentration following a power law $G^{\prime}, G^{\prime \prime} \infty \phi^{b}$ with exponent $b \sim 2$ (see Fig. 6).

2. The critical stress $\sigma_{y}$ at which the samples start to show nonlinear elastic response increases linearly with the polymer concentration $\sigma_{y} \propto \phi^{c}$ with $c \sim 1$.

3. The critical strain linearly decreases as the polymer concentration increases $\gamma_{y} \propto \phi^{d}$ with $d \sim 1$. 


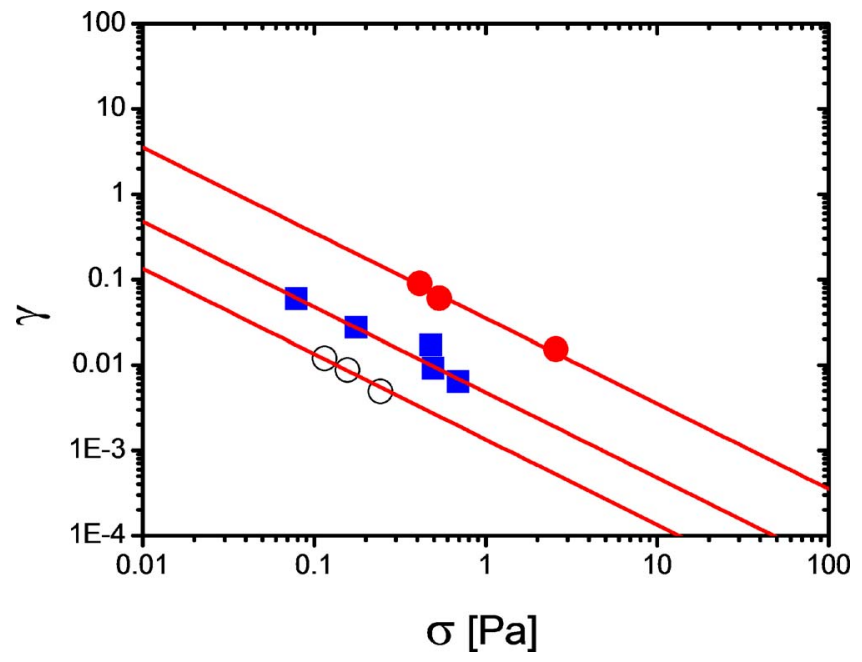

FIG. 8. Critical strain $\gamma_{y}$ as a function of critical stress $\sigma_{y}$ for three samples at different salt and polymer concentrations. Squares refer to the set with $200 \mathrm{mM} \mathrm{NaCl}$ and polymer concentration ranging from $0.05 \%$ to $0.4 \%$ (compare with Fig. 7). Solid circles refer to the sample at $100 \mathrm{mM} \mathrm{NaCl}$ and open circles to the set with $300 \mathrm{mM} \mathrm{NaCl}$. Lines are the best hyperbolic fit to the three data sets.

The same scaling of the rheological properties is found for three different salt concentrations. The elastic modulus at small deformation increases and both critical stress and strain decrease with increased salt concentration.

The scaling found for the elastic modulus differs from literature results for QC samples [Morris et al. (1999)] in which the elastic modulus scales with polymer concentration to the third power. Moreover, the critical strain is typically reported as independent of the polymer concentration [Ketz et al. (1988); Morris et al. (1999)], again distinct from our results on SC systems. Since the essential difference between SC and QC samples is their microstructure, it is useful to compare our bulk rheology results with those found for concentrated microgel particle suspensions [Adams et al. (2004)]. The most striking agreement between the results of Adams et al. and our SC gel is the scaling of the critical strain as a function of the polymer concentration. For the case of agar microgel particles at fixed volume fraction, increased polymer concentration in each microgel particle leads to a linear reduction of the critical strain, similar to what we find in our SC gellan gum samples. The comparison between these two systems is even more convincing if we consider the variation of the agar elastic modulus at fixed volume fraction while the single particle polymer concentration is varied: they find a power law scaling of the modulus with an exponent of about 2, similar to our SC samples.

In our gellan gum system, we lack the ability to control the polymer concentration in a single microgel particle, but we conclude from optical microscopy that the deformable microgel particles' volume fraction is constant in our samples and above the spherical close-packing value [Adams et al. (2004)]. Thus, in our system an increased polymer concentration directly impacts the single particle rheological properties.

We also report an additional surprising experimental result, which certainly needs more investigation and could help lead to a better understanding of the yielding process of such microstructured systems. Robertson and Wang (2005) recently found the shearinduced jammed-unjammed transition to be isoenergetic for silica dispersed in oil at several volume fractions. As a result, the mechanical energy input required to unjam 
(yield) the system, defined as the product of $\sigma_{y}$ and $\gamma_{y}$, is constant with volume fraction and can be considered as a critical energy density required to unjam the system. This characteristic has been found in other systems like filled rubber [Wang and Robertson (2005)], boehmite alumina gel [Shih et al. (1990)], and concentrated suspensions of microgel particles [Adams et al. (2004)]. If we interpret the yielding of our SC samples as a shear-induced transition from a jammed state to an unjammed state, we find the same isoenergetic character, since the product $\sigma_{y} \gamma_{y}\left[\mathrm{~J} / \mathrm{m}^{3}\right]$ remains constant at constant salt concentration. Increased salt concentration instead reduces the critical energy required to unjam the system.

\section{CONCLUSIONS}

The results presented here are important for optimizing the rheological properties of a gellan gum hydrogel system. We clarify the structural difference between QC and SC gels, and we characterize local gel elasticity by means of microrheology. Our results confirm a previous study on shear effects on biopolymer gelation and provide a more quantitative understanding of microrheology and the relation between microstructure and bulk elastic properties.

Increased insight into shear effects on gel microstructure may enable improved control of structured suspension formulations requiring precise tuning of both linear and nonlinear rheological properties. SC gellan gum systems allow such control by fine tuning polymer and salt concentration to access a wide range of elastic properties at small deformations while also controlling the yield behavior. Further investigation is needed to quantify shear's role in determining the characteristic gel microstructure size. This is an essential step in developing a quantitative predictive model of the structural properties of a SC gel based on its composition and preparation. Finally, our interpretation of the SC gel rheology in the context of a jamming transition appears reasonable but its underlying origin needs further investigation.

\section{ACKNOWLEDGMENTS}

The authors acknowledge support from the Procter \& Gamble Company, the Harvard MRSEC (DMR-0213805) and the A. Della Riccia Foundation.

\section{References}

Adams, S., W. J. Frith, and J. R. Stokes, "Influence of particle modulus on the rheological properties of agar microgel suspensions," J. Rheol. 48, 1195-1213 (2004).

Altmann, N., J. J. Cooper-White, D. E. Dunstan, and J. R. Stokes, "Strong through to weak 'sheared' gels," J. Non-Newtonian Fluid Mech. 124, 129-136 (2004).

Crocker, J. C., and D. G. Grier, "Methods of digital video microscopy for colloidal studies," J. Colloid Interface Sci. 179, 298-310 (1996).

Ketz, R. J., R. K. Prud-homme, and W. W. Graessley, "Rheology of concentrated microgel solutions," Rheol. Acta 27, 531-539 (1988).

Liu, J., M. L. Gardel, K. Kroy, E. Frey, B. D. Hoffman, J. C. Crocker, A. R. Bausch, and D. A. Weitz, "Microrheology Probes Length Scale Dependent rheology," Phys. Rev. Lett. 96, 118104 (2006).

Mason, T. G., "Estimating the viscoelastic moduli of complex fluids using the generalized Stokes-Einstein equation," Rheol. Acta 39, 371-378 (2000).

Miyazaki, K., H. M. Wyss, D. A. Weitz, and D. R. Reichman, "Nonlinear viscoelasticity of metastable complex fluids," Europhys. Lett. 75, 915-921 (2006). 
Morris, E. R., R. K. Richardson, and L. E. Whittaker, "Rheology and gelation of deacylated gellan polysaccharide with $\mathrm{Na}^{+}$as the sole counterion," Prog. Colloid Polym. Sci. 114, 109-115 (1999).

Norton, I. T., D. A. Jarvis, and T. J. Foster, "A molecular model for the formation and properties of fluid gels," Int. J. Biol. Macromol. 26, 255-261 (1999).

Ogawa, E., R. Takahashi, H. Yajima, and K. Nishinari, "Thermally induced coil-to-helix transition of sodium gellan gum with different molar masses in aqueous salt solutions," Biopolymers 79, 207-217 (2005).

Ohtsuka, A., and T. Watanabe, "The network structure of gellan gum hydrogels based on the structural parameters by the analysis of the restricted diffusion of water," Carbohydr. Polym. 30, 135-140 (1996).

Oppong, F. K., L. Rubatat, B. J. Frisken, A. E. Bailey, and J. R. de Bruyn, "Microrheology and structure of a yield-stress polymer gel,” Phys. Rev. E 73, 041405 (2006).

Robertson, C. G., and X. R. Wang, "Isoenergetic jamming transition in particle-filled systems," Phys. Rev. Lett. 95, 075703 (2005).

Shih, W. H., W. Y. Shih, S. Kim, J. Liu, and I. A. Aksay, "Scaling behavior of the elastic properties of colloidal gels," Phys. Rev. A 42, 4772-4779 (1990).

Sworn, G., G. R. Sanderson, and W. Gibson, "Gellan gum fluid gels," Food Hydrocolloids 9, 265-271 (1995). Valentine, M. T., P. D. Kaplan, D. Thota, J. C. Crocker, T. Gisler, R. K. Prud-homme, M. Beck, and D. A. Weitz, "Investigating the microenvironments of inhomogeneous soft materials with multiple particle tracking," Phys. Rev. E 64, 061506 (2001).

Wang, X. R., and C. G. Robertson, "Strain-induced nonlinearity of filled rubbers," Phys. Rev. E 72, 031406 (2005). 